\title{
Milk of calcium in urethral diverticulum in a male patient with paraplegia and suprapubic urinary drainage
}

\author{
Spinal Cord (2004) 42, 57-58. doi:10.1038/sj.sc.3101540
}

Milk of calcium contains a colloidal suspension of calcium crystals and is found commonly in certain locations of the urinary tract where drainage from the cavity is less than optimum, for example pyelocalyceal diverticulum or, in the inferior calyx of a poorly functioning, hydronephrotic kidney. ${ }^{1}$ Stasis and urinary tract infections are the most important aetiologic factors in the occurrence of milk of calcium. ${ }^{2}$ In addition to stasis and infection, gravity and physical immobility may play a vital role in the pathogenesis of milk of calcium, particularly in spinal cord injury (SCI) patients. Gravity has been shown to contribute to formation of renal calculi. A prospective study on patients with recurrent unilateral nephrolithiasis revealed that the sleep posture correlated with the side of stone recurrence. $^{3}$ Of the 110 patients with recurrent unilateral nephrolithiasis, 93 slept consistently with one side in a dependent position and the side of stone was identical to the dependent sleep side in $76 \%(P=0.008)$.

SCI patients with ischial or perineal pressure sores are often advised bed rest for prolonged periods. With imposed restrictions on physical activity and mobility, these patients can become vulnerable to develop milk of calcium in the urinary tract. We report a male patient with paraplegia in whom, milk of calcium was detected in a urethral diverticulum by computed tomography.

A 19-year-old male sustained paraplegia at T-4 level in a motor bike accident in 1966 . He wore penile sheath and emptied the bladder by expression. Over the years, he developed a large urethral diverticulum at the penoscrotal junction. In June 2001, he noticed urine leak from perineal pressure sore, which he had for 15 months. Therefore, suprapubic cystostomy was performed and urine leak from the sore stopped. He was advised bed rest to facilitate complete healing of perineal and left ischial pressure sores. In July 2002, he was still not getting up from bed because of pressure sores. Intravenous urography showed bilateral hydronephrosis and hydroureter. CT of abdomen showed bilateral hydronephrosis. No mass lesion was seen in the bladder. An incidental observation in CT of pelvis was a dependent, high-attenuating material with a horizontal upper edge in the anterior part of an hourglass-shaped urethral diverticulum (Figure 1). This specific radiological finding in the CT provided a clue that the highattenuating material with a horizontal upper edge represented milk of calcium in the urethral diverticulum. The CT attenuation number of 'milk of calcium' was

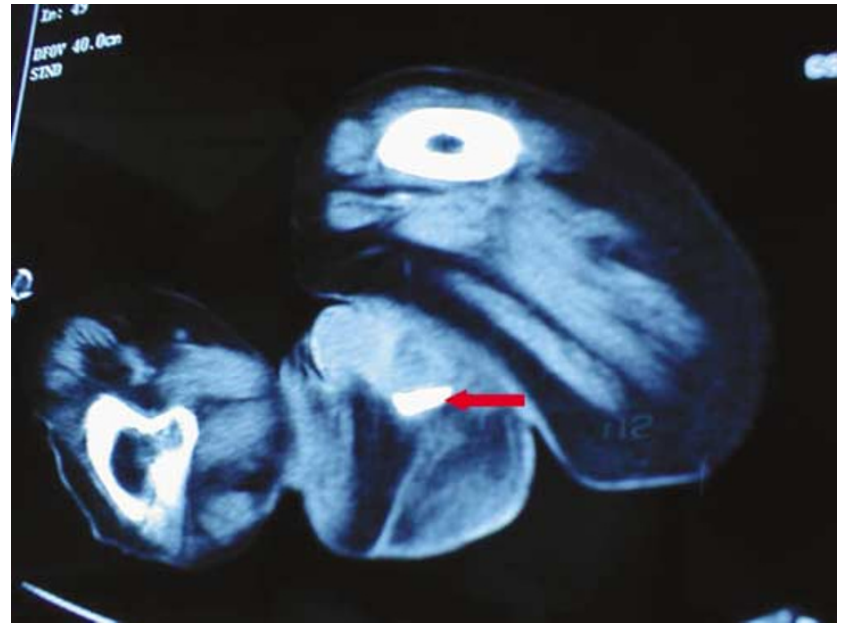

Figure 1 Axial CT of pelvis in a patient with SCI and paraplegia at T-4 level: the dependent, high-attenuating material, which has a horizontal upper edge, is located in the anterior part of an hourglass-shaped urethral diverticulum (arrow). The CT attenuation number is 493, which indicates calcium

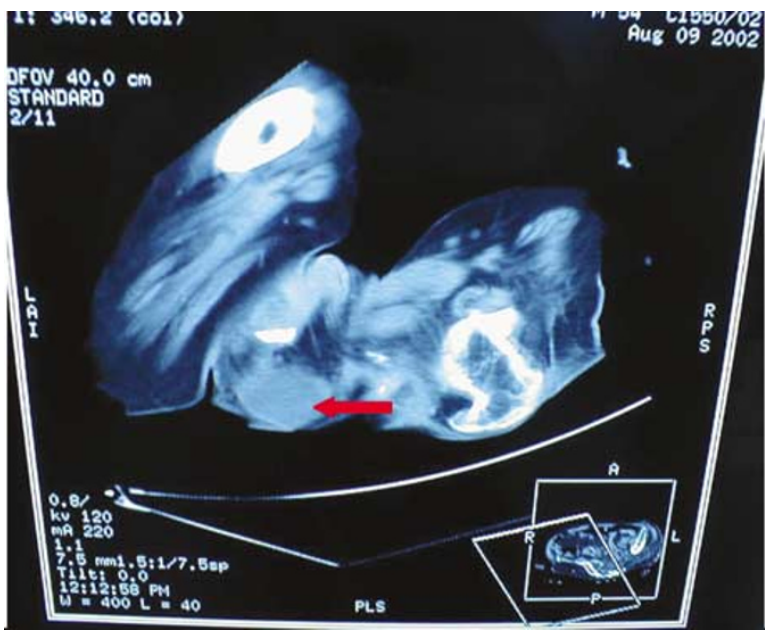

Figure 2 Oblique axial reformatted image of CT of pelvis shows both components of hourglass-shaped urethral diverticulum. The high-attenuation material with a horizontal upper edge is present in the anterior part; the posterior part of the hourglass-shaped diverticulum contains low-attenuating fluid (arrow) 
493. The posterior part of the hourglass-shaped contained low-attenuating fluid (Figure 2).

Flexible cystoscopy showed debris and sediment in the diverticulum. There was no formed calculus, thus confirming the diagnosis of milk of calcium in urethral diverticulum. The contents of urethral diverticulum were washed out during flexible cystoscopy. No tumour was seen in the diverticulum or in the urinary bladder.

Subsequently, biopsies of bladder mucosa, taken with a rigid cystoscope, revealed vesical malignancy. This patient underwent radical cystectomy and ileal conduit urinary diversion. Histology showed transitional cell carcinoma with extensive squamous and glandular differentiation, extending through full thickness of bladder wall into perivesical fat.

In this SCI patient, presence of dependent, highattenuating material with a horizontal upper edge in CT of pelvis was diagnostic of milk of calcium in urethral diverticulum. The predisposing factors for development of milk of calcium in the urethral diverticulum were:

- Absence of natural flow of urine per urethra because of suprapubic urinary diversion.

- Restrictions imposed on physical activity and mobility due to prolonged periods of bed rest (to facilitate healing of perineal and ischial pressure sores).

- Recurrent urinary infections largely due to long-term indwelling catheter drainage.

Although milk of calcium is often a benign condition, sometimes, complications such as stone formation or infection may occur. ${ }^{4}$ If SCI patients with urethral diverticulum have undergone only urinary diversion, the diverticulum requires washings at regular intervals to get rid of debris and urinary sediment. Such a procedure may be comparable to irrigation of defunctionalised neuropathic bladder recommended for SCI or spina bifida patients, who have undergone supravesical urinary diversion.

$$
\begin{array}{r}
\text { S Vaidyanathan }{ }^{1}, \mathrm{PL} \text { Hughes }^{2}, \mathrm{BM} \mathrm{Soni}^{1}, \mathrm{G} \mathrm{Singh}^{1}, \\
\mathrm{P} \text { Mansour }{ }^{3} \text { and P Sett }{ }^{1} \\
{ }^{1} \text { Regional Spinal Injuries Centre } \\
\text { District General Hospital, Southport PR8 6PN, UK; } \\
{ }^{2} \text { Department of Radiology, District General Hospital, } \\
\text { Southport PR8 } 6 P N, U K \\
\text { and }{ }^{3} \text { Department of Cellular Pathology } \\
\text { District General Hospital, Southport PR8 } 6 P N, U K
\end{array}
$$

\section{Acknowledgements}

Our gratitude to $\mathrm{Mr}$ Brian Grimes, Product Group Manager-OPM, B. Braun Medical Ltd, Chapeltown, Sheffield, S35 2PW, United Kingdom, for sponsoring the printing of the colour illustrations.

\section{References}

1 Vaidyanathan S et al. Milk of calcium in the inferior calyx of a hydronephrotic kidney in a tetraplegic patient - a diagnosis to be made before scheduling for extracorporeal shock wave lithotripsy. Spinal Cord 2000; 38: 325-326.

2 Garcia-Cuerpo E et al. New location of milk-of-calcium. Urology 1985; 25: 425-427.

3 Shekarriz B, Lu HF, Stoller ML. Correlation of unilateral urolithiasis with sleep posture. J Urol 2001; 165: 1085-1087.

4 Healey T, Way BG, Grundy WR. Milk of calcium in calycine diverticula. Br J Radiol 1980; 53: 845-852. 